\title{
La reconfiguración del régimen financiero global: Entre la determinación pública y la autodeterminación privada
}

\author{
The Reconfiguration of the Global Financial Regime: Between the \\ Public Determination and the Private Self-determination
}

Benjamín Sánchez

Facultad de Ciencias Sociales, Universidad Andrés Bello, Chile

\begin{abstract}
RESUMEN
La crisis financiera del 2008 modificó las relaciones sostenidas entre el sistema político, la esfera jurídica y el dominio financiero a escala global. Ante la incapacidad de las estructuras regulatorias de los países afectados por salvaguardar la estabilidad de sus mercados, la solución política ha estribado en un incremento de la participación estatal sobre los órganos jurídicos encargados de supervigilar los procesos financieros, provocando un nuevo giro en la disputa por el control de la regulación financiera sostenida entre el sistema político y el sistema financiero. Este artículo indaga los orígenes y los potenciales efectos de esta dinámica emergente, identificando los principales acoplamientos estructurales dispuestos por el Estado para subsanar los vicios funcionales del régimen financiero global, así como las raíces de la desdiferenciación derivada de la captura regulatoria en la que ha incurrido históricamente el sistema financiero. Para describir este fenómeno desde la perspectiva sociológica, se conjugó la óptica analítica que proporciona la teoría de sistemas con el arsenal teórico propio de la regulación financiera, incluyendo una investigación empírica en dicho campo. Finalmente, este artículo propone resaltar las ventajas funcionales de esta pugna intersistémica, introduciendo una reflexión que rescata sus beneficios para la sociedad en el largo plazo.
\end{abstract}

PALABRAS ClAVE: Regímenes regulatorios; Sociología jurídica; Sistema financiero; Desdiferenciación; Teoría de sistemas

\section{ABSTRACT}

The financial crisis of 2008 amended the sustained relationships between the political system, the legal system and the financial system globally. Given the inability of the regulatory structures of the concerned countries to safeguard the stability of its markets, the political solution has focused in increased state involvement on legal bodies to supervise financial processes, causing a new turn in the dispute over sustained control of financial regulation between the political system and the financial system. This article explores the origins and the potential effects of this emerging dynamics, identifying the main structural links arranged by the State to correct the functional defects of the global financial system, as well as the roots of dedifferentiation derived regulatory capture in which incurred historically the financial system. To describe this emerging dynamics from a sociological perspective, was conjugated the optic that provides analytical systems theory with own theoretical arsenal of financial regulation, including recent empirical research in this field. Finally, this article highlight the functional advantages of this intersystemic struggle, introducing a reflection that rescues its benefits to the social system in the long term.

KEYWORDS: Regulatory regimes; Legal sociology; Financial system; Dedifferentiation; Systems theory

REVISTA MAD - UNIVERSIDAD DE CHILE

Revista del Magíster en Análisis Sistémico Aplicado a la Sociedad

Facultad de Ciencias Sociales, Departamento de Antropología.

Universidad de Chile

www.revistamad.uchile.cl 


\section{INTRODUCCIÓN*}

La severa crisis que sacudió los mercados financieros el 2007-2008 sirve como caso paradigmático para ilustrar cómo la progresiva complejización del sistema social puede redundar en un caso de disfunción multifactorial. Por dicha razón, resultan sesgado identificar en un sólo proceso sistémico los diversos y enmarañados orígenes de la desestabilización. En este sentido, la debacle se engendró por una mezcla de disfunciones tanto en el plano intra-sistémico, como en el contexto inter-sistémico.

Sobre las causas endógenas, se conjugaron una serie de factores patológicos que socavaron la estabilidad de una relevante parte del mercado financiero mundial. Entre ellas destaca la compulsión de dicho sistema por crecer desmesuradamente en su producción de dinero (Teubner 2011), lo cual condujo a una inevitable sobre-extensión de sus procesos inherentes y a la consiguiente ruptura de la burbuja inmobiliaria en EEUU (Kjaer 2011). A ello se sumó la incapacidad de sus mecanismos cognitivos para evaluar y anticipar sus riesgos intrínsecos (Ladeur 2011), como consecuencia de una sobre-cognitivización de sus estructuras. Estas desestabilizaciones se potenciaron con la profusa interconexión entre los mercados domésticos, regionales e intercontinentales, cuyos enlaces permitieron propagar las perturbaciones a lo largo del sistema (Stäheli 2011).

De forma exógena, el progresivo eclipse del Estado ha devenido en una subsecuente inestabilidad de las estructuras que articulan a las esferas jurídicas, financieras y políticas (Febbrajo 2011), provocando un fallo en las funciones regulatorias del sistema jurídico, encargado de fiscalizar el correcto desenvolvimiento de los procesos financieros tanto en el plano doméstico, como a escala regional y en el estrato de la misma funcionalidad financiera, aunque por motivos dispares y con efectos peculiares para cada nivel.

Este raquitismo estatal, demostrado en algunas jurisdicciones nacionales, pavimentó el camino a una "desdiferenciación" entre ámbitos autónomos como el derecho y la economía, expresada en entidades regulatorias domésticas sumamente permisivas, y en agendas internacionales igualmente insuficientes. La reacción inmunológica por parte del sistema

\footnotetext{
* Este texto buscó contribuir como aporte teórico al proyecto Fondecyt N1110437, titulado "Colisión y armonización de regímenes regulatorios en contextos sociales globales", conducido por los académicos Elina Mereminskaya y Aldo Mascareño. Agradezco a este último por brindarme la oportunidad de participar en dicha investigación, así como por las fructíferas discusiones y los valiosos consejos que me proporcionó durante la confección del presente artículo. Extiendo mis agradecimientos también a Alejandro Espinosa, en razón de su permanente presencia y orientación en los momentos claves.
} 
social, se ha expresado en la creación de nuevos acoplamientos estructurales entre la política y el derecho, con el motivo de aumentar la influencia de los actores gubernamentales en los cuerpos regulatorios del sistema jurídico (Pagliari 2012a, Gadinis 2013a).

No obstante, existe una diferencia sustancial entre los procesos de politización y de estatización. Para comprender la distancia semántica entre ambos conceptos se debe partir de ciertas premisas. Primero que todo, la política constituye un sistema capaz de ofrecer sus operación relativa a la toma de decisiones colectivamente vinculantes (Luhmann 2006), a los diversos sistemas que lo requieran. Por lo mismo, a pesar de que tradicionalmente se ha reconocido a la figura del Estado Nación como el modelo estructural por excelencia de lo político (Mereminskaya \& Mascareño 2005), este no se reduce únicamente a lo estatal (Teubner 1997). Como ejemplo de esto para el caso del sistema financiero, los mismos individuos que activan sus procesos también pueden desarrollar dinámicas remitidas a lo político, por medio de su interacción y correspondiente institucionalización como stakeholders (Kjaer 2011). Este término surge como equivalente semántico al concepto de "pueblo", significando un avance evolutivo al englobar a actores afectados por la actividad de determinado sistema social, sin distinción por su adscripción a un territorio específico o por su pertenencia a determinada cultura, posibilitando así la participación en la toma de decisiones (es decir, su consideración por parte del sistema político) en el espacio transnacional.

Por ende, la reestructuración de la arquitectura financiera doméstica tal y como se describe en este artículo, corresponde a una dinámica en la cual se enfrentan dos movimientos contrapuestos de politización. De un lado se encuentran los esfuerzos gubernamentales por estatizar la regulación financiera, mientras que en la otra orilla residen los intentos de las mismas instituciones y actores destinatarios de la regulación por moldearla conforme a sus propios intereses (Polanyi 2001). Por lo mismo, así como es posible hablar de actores políticos de carácter privado, también resulta consistente identificar actores financieros de índole pública, como sucede en el caso del fisco.

Ahora bien, el origen de toda crisis puede ser rastreado en un vacío de conocimiento. Si bien cada sistema social es capaz de producir información en base a su propio aparato cognitivo, las descripciones científicas manifiestan una consistencia epistemológica superiores a las de las demás 
esferas sociales $^{1}$ (Luhmann 2005). Por lo mismo, indagar e identificar los puntos débiles del dique que mantiene a raya el torrente de disrupciones financieras no posee valor sólo para las ciencias sociales -y para la disciplina sociológica en este caso-, sino que también contribuye al resguardo de la integridad social, y más aún, cuando la función del sistema financiero ha demostrado tener una relevancia radical para los demás sistemas que dependen de sus operaciones.

Para describir esta dinámica emergente desde una perspectiva sociológica, se conjugó el arsenal teórico que proporciona la teoría general de sistemas sociales, con bibliografía académica propia de la regulación financiera, privilegiando las obras surgidas con posterioridad a la crisis. Como complemento a dichas fuentes secundarias, se utilizaron los resultados estadísticos proporcionados por Stavros Gadinis (2013a), para analizar las recientes reformas emprendidas en el campo de la regulación financiera doméstica.

En lo que respecta a la estructura temática del presente artículo, en la primera sección se define el concepto de régimen regulatorio financiero, junto con una descripción de sus componentes básicos (1). Acto seguido, quedan plasmadas las vicisitudes que asechan a la regulación financiera doméstica, producto de los procesos de desdiferenciación que ejecuta el sistema financiero para capturar su infraestructura jurídica (2). Posteriormente, se detallan las reformas regulatorias tanto a nivel doméstico como a escala transnacional, junto con un análisis de los potenciales efectos de desdiferenciación política implicados en esta dinámica (3). Para concluir, se sintetizan las modificaciones del paisaje regulatorio a escala global, finalizando con una evaluación funcional de la dialéctica sostenida entre la regulación pública y la autodeterminación privada.

\section{PROPIEDADES GENERALES DEL RÉGIMEN FINANCIERO GLOBAL}

La función del sistema jurídico es la de asegurar expectativas normativas (Luhmann 2005), lo cual equivale a promover la certeza de que si determinado comportamiento codificado normativamente es transgredido, se tomarán medidas para restituir, compensar el daño, o sancionar el crimen

\footnotetext{
${ }^{1}$ En la medida que su medio simbólicamente generalizado, la verdad, ejecuta un proceso de doble reflexividad por medio del cual la verdad se vuelca sobre sí misma: la verdad define qué hay de verdadero en ella. Esta peculiaridad se traduce en la creación y el refinamiento de métodos para definir qué es cierto y qué no lo es, lo que en ningún caso significa que el conocimiento generado por el resto de las esferas sociales sea inválido. Sin embargo, la ciencia asegura filtros procedimentales más rigurosos al momento de generar heterodescripciones.
} 
cometido. Es por esto que el centro institucional de la comunicación jurídica emana desde los tribunales, dado que allí se genera la instancia para que el derecho aplique su código elemental basado en la distinción de los valores derecho/no-derecho. No obstante, en la periferia del derecho es posible identificar la elaboración de estructuras normativas como protocolos, reglamentos y marcos regulatorios (Teubner 2012).

Como señala Mascareño (2007: 3), el derecho se despliega de manera especial en cada uno de los niveles de la sociedad mundial, a la cual entiende como la "articulación emergente de los niveles territorial, regional y global", lo cual permite hablar de cuatro tipos de derecho: el nacional (a), el internacional (b), el supranacional (d) y el transnacional (e); formas jurídicas que al entrar en contacto con los requerimientos funcionales del sistema financiero dan vida al régimen financiero y lo segmentan en estratos estrechamente imbricados. Antes de proseguir, es menester detallar algunos aspectos esenciales en torno a la composición estructural de cada nivel, teniendo como referencia el régimen financiero.

a) El derecho nacional engloba a todas aquellas formas de producción legislativa dentro de las fronteras delimitadas estatalmente, que para efectos del régimen financiero, se expresan en las agencias regulatorias nacionales ${ }^{2}$, tales como la Securities and Exchange Commission (SEC) para el caso de EEUU, o en el caso de Alemania, la Autoridad Federal de Supervisión Financiera (BaFin por sus siglas en alemán). En algunos países, los bancos centrales albergan a las respectivas agencias regulatorias dentro de sus dependencias, mientras que en otras jurisdicciones nacionales, los mismos bancos centrales cargan con dicha función regulatoria (Seeling \& Novoa 2009).

b) El derecho internacional, por su parte, remite a formas de cooperación multilateral entre países (Mascareño 2013), reconocibles en los organismos financieros resultantes del acuerdo de Bretton Woods en 1944, llámese el Fondo Monetario Internacional (FMI) o el Banco Mundial (BM). En esta forma de derecho también se incluyen arreglos e instituciones financieras de índole regional, evidenciadas en la pluralidad de entidades regulatorias europeas, como el Sistema Europeo de Supervisores Financieros (SESF) o el Consejo Europeo de Riesgo Sistémico (CERS) (Lo Schiavo 2013).

\footnotetext{
${ }^{2}$ Descritas en profundidad en la sección 1.
} 
c) El derecho supranacional se refiere a aquellas instancias jurídicas en las cuales los estados delegan su autonomía soberana a un tribunal calificado, cuyo fallo determina consecuencias vinculantes para ambas partes (Mascareño 2013). Probablemente, la institución supranacional más destacable del régimen financiero corresponde al Centro Internacional de Arreglo de Diferencias Relativas a Inversiones (CIADI), cuya misión estriba en arbitrar litigios y dictaminar laudos para la resolución de controversias.

d) Finalmente, en el nivel global de la sociedad, aparece el derecho transnacional como consecuencia del progresivo aumento en la complejidad de cada sistema social, lo que ha conllevado a un incremento de las contingencias a las que éstos han debido responder (Luhmann 2006). Para evitar que las expectativas emergentes devengan en masivas desilusiones individuales, y que éstas engendren consiguientes disrupciones sociales, los sistemas laterales mundiales -llámese el conjunto de actores, organizaciones e instituciones transnacionales que activan la comunicación de cada esfera social (Willke 2007)- han recurrido al sistema del derecho para generar soportes normativos que corrijan posibles conflictos externos e internos, al encauzar sus procesos inherentes dentro de parámetros seguros (Teubner 1997).

El derecho financiero transnacional, estuvo compuesto hasta fines de los 90 por dos tipos de instituciones, categorizadas dependiendo de su carácter semipúblico (d.1) o netamente privado (d.2). Sin embargo, existe una base infraestructural que escapa a aquella tipología propuesta (d.3), cuyas peculiaridades que serán explicitadas en lo sucesivo.

d.1) Ante la descrita ausencia de directrices que orienten las contingencias de los actores financieros, dichos vacíos regulatorios han intentado ser cubiertos por el mismo sistema financiero con la asistencia del sistema jurídico, dando lugar a organizaciones regulatorias sin pertenencia o raíz estatal alguna. En esta clasificación se incluyen las agencias de calificación de riesgo (conocidas por el oligopolio que conforman Moody's, Standars and Poors y Fitch), las organizaciones de estandarización contable (como el Financial Accounting Standars Board (FASB)) y otras organizaciones sectoriales como la Asociación Internacional de Swaps y Derivados (ISDA). 
d.2) De forma paralela, las organizaciones de derecho transnacional privado coexisten con una pluralidad de organismos regulatorios semipúblicos, los cuales se encuentran compuestos por la agrupación voluntaria de las diversas entidades supervisoras domésticas. En un afán de mejorar y armonizar tanto las prácticas como los marcos regulatorios producidos por las agencias regulatorias nacionales, se ha generado una forma de derecho transnacional híbrida, categoría que engloba a instituciones como el Comité de Basilea de Supervisión Bancaria (BCBS), la Organización Internacional de Comisiones de Valores (OICV) o la Asociación Internacional de Supervisores de Seguros (IAIS), entre otras.

d.3) En abril de 1999, los ministros de finanzas y los gobernadores de los bancos centrales del G7 deciden crear un organismo que reúna y coordine a las instituciones regulatorias más relevantes del régimen financiero global. Como resultado, nace el Foro de Estabilidad Financiera (o Financial Stability Forum (FSF) por sus siglas en inglés), cuya función es la de servir como punto de reunión y colaboración entre las entidades supervisoras domésticas, los organismos financieros internacionales (como el foro del G10 originalmente), el Banco Central Europeo para el caso regional, y diversas instituciones regulatorias transnacionales privadas e híbridas (Gadinis 2013b). A través del FSF, la pluralidad de instituciones regulatorias que componen el régimen financiero puede incrementar el flujo de información relevante entre ellas, atributo que se complementa con la proporción de instancias para la observación mutua, lo que facilitan la búsqueda de un consenso hacia metas comunes.

En relación con la transnacionalización del derecho financiero, se ha establecido un paralelo entre la creación de estatutos sectoriales globales y el proceso de constitucionalización propio de los estados nación (Teubner 2012). De este modo, se plantea que el derecho se ha fragmentado en una constelación de constituciones de carácter civil representativas de las distintas esferas sociales, lo cual se ha materializado en la aparición de una genuina lex financiaria para el caso del subsistema financiero. No obstante, dado que la función principal de estas constituciones sui generis es la de regular dominios sistémicos particulares, Mereminskaya y Mascareño 
(2011) proponen hablar de regímenes regulatorios, concepto que será incorporado y adaptado para efectos del presente artículo ${ }^{3}$.

Puesto que el objeto de este artículo no se restringe a la indagación de las características estructurales del derecho supranacional o transnacional ligado a lo financiero, la perspectiva analítica que ofrece el concepto de regímenes regulatorios resulta insatisfactoria si no se readecúa y expande su foco descriptivo. En adelante, se hablará de régimen financiero para hacer referencia al sector específico del sistema jurídico encargado de velar por la regulación del sistema financiero, como zona de contacto entre derecho y finanzas ${ }^{4}$.

De cierto modo, resulta equivalente al término "arquitectura financiera", recurrente en la jerga jurídico-económica (Armijo 2001). Sin embargo, aquel concepto no diferencia con suficiente claridad las estructuras financieras de las jurídicas, distinción crucial desde la lógica teórica de índole sistémico. En cambio, el concepto de régimen regulatorio presupone que ambos planos se encuentran clausurados operativamente, pero enlazados y acoplados institucionalmente.

Así como la semántica "persona", alude a una construcción simbólica que brota del acoplamiento estructural entre un sistema biológico particular (cuerpo humano) con un determinado sistema psíquico (conciencia) (Luhmann 2006), sostengo que la morfología del régimen financiero global posee una identidad eminentemente dual. Desde dicha perspectiva, el régimen se puede observar y describir tanto desde un punto de vista cartográfico (a) como de uno radiográfico (b).

a) En lo que respecta al nivel superficial su composición, el régimen se encuentra conformado infraestructuralmente por una pluralidad de instituciones privadas, públicas y semipúblicas que permiten disminuir los costos de transacción implicados en la elaboración de outputs regulatorios (Willke 2007).

b) Trasladándonos al segundo nivel, la coordinación jerárquica de los actores que integran la multiplicidad de entidades regulatorias sirve de catalizador para la emergencia de conjunto diversifi-

\footnotetext{
${ }^{3}$ De todas maneras, las diferencias entre ambas semánticas son poco significativas, por lo que no resulta inconsistente hablar de regímenes regulatorios y de constituciones sociales a la vez.

${ }^{4}$ Para el caso particular del régimen financiero, éste carece de un organismo central desde el cual emane normatividad con carácter vinculante, como sucede con la Organización Mundial del Comercio (OMC) en lo que atañe a la lex mercatoria (Mereminskaya \& Mascareño 2011). Más bien, el régimen está compuesto por una red de instituciones regulatorias especializadas en un sector estructural específico del sistema financiero (Douglas \& Taylor 2009).
} 
cado de normas y procedimientos (medios) que se vinculan, agrupan y condensan en una serie de dispositivos de corrección y mecanismos preventivos (formas) con la finalidad de salvaguardar, no sólo la estabilidad del sistema financiero, sino también de acelerar y extender su actividad intrínseca.

La configuración programática del régimen financiero ha oscilado a lo largo de su historia entre dos principios contrapuestos, basados en la dicotomía regulación pública/autorregulación privada. Karl Polanyi (2001) describió en extenso el desenvolvimiento de tal dinámica hasta mediados del siglo XX, sintetizándola en el célebre concepto de "doble movimiento". La pasada crisis marcó un nuevo vuelco en el vaivén programático del régimen financiero, lo cual se ha expresado en una intensificación de la presencia estatal en las estructuras financieras, luego de años de ver cooptada su autonomía operativa por parte del sistema financiero.

2. DESDIFERENCIACIÓN DIRIGIDA POR EL SISTEMA FINANCIERO: LA VULNERABILIDAD DE LA REGULACIÓN FINANCIERA DOMÉSTICA

El primer ladrillo sobre el que se erige toda la arquitectura infraestructural del régimen radica en las agencias regulatorias nacionales, definidas como organizaciones públicas de carácter autónomo, lo que las sitúa a mitad de camino entre el mundo público y la dimensión privada. Eric Pan (2011: 1909) identifica su principal función en "el diseño, la promulgación y en la ejecución de la regulación", para lo cual el sistema político les delega facultades ejecutivas de producción normativa (Amstutz 2011), responsabilizándolas además por la fiscalización de aquellos marcos regulatorios.

Según lo planteado por Pan (2011), las entidades regulatorias domésticas se ven condicionadas a optar entre dos tipos de estrategias opuestas para efectuar sus labores regulatorias, las que se categorizan taxonómicamente en estrategias públicas y privadas; ambas pueden reconocerse, en términos sistémicos, como dos configuraciones programáticas distintas.

La programación pública se traduce en inputs directos del organismo regulatorio en su entorno institucional, mediante los cuales se exige a los participantes del mercado adscribirse a las reglas prudenciales dispuestas. Asimismo, efectúan labores exhaustivas de supervisión y ejecución que complementan con rigurosas evaluaciones y certificaciones. 
Los reguladores nacionales también pueden elegir realizar una descarga operativa hacia las mismas instituciones financieras, abogando por la autorregulación del sistema financiero en detrimento de una forma de monitoreo externo. Para transferir dicha responsabilidad, se fijan principios abocados a la divulgación de la información interna de cada organización hacia el mercado, a modo de aumentar la transparencia operativa ${ }^{5}$. Junto a ello, se potencia la creación de instituciones autorreguladoras (self-regulatory institutions (SROs)), entendidas como asociaciones financieras gremiales encargadas de producir protocolos y estándares para su sector específico (crediticio, de inversión, previsional, etc...), entre otras medidas como la asistencia de gatekeepers u oficiales internos de cumplimiento.

A pesar de lo que se pudiese concluir a primera vista, la programación pública no representa necesariamente los intereses del sistema político. Puesto que el gobierno de turno puede tener dentro de sus planes una política fiscal expansiva, una fórmula conveniente podría ser permitir mayor concesión de créditos y, por ende, promover una regulación que permita a los bancos tomar más riesgos. Por esta razón, la autocorrección del sistema financiero puede ser el programa regulatorio más adecuado para acelerar su rendimiento en el corto plazo, lo que trae consigo un desmedro en la estabilidad de sus procesos.

Según Pan (2011), la elección de uno u otro enfoque depende principalmente de los recursos con los que cuente cada entidad supervisora. No obstante, como se ha anticipado al respecto, la apropiación de la infraestructura regulatoria por parte del aparato estatal y de los actores financieros privados, torna aún más enmarañada la difusa naturaleza sociofuncional de las organizaciones regulatorias.

A pesar de que el código operativo que orienta las comunicaciones regulatorias confirma el rol de las entidades supervisoras como un engranaje más en la maquinaria del derecho (Febbrajo 2011), éste subsistema parcial debe echar mano a conocimientos propiamente financieros para comprender las semánticas y los procesos de aquel dominio. Con la finalidad de interpretar lo que acontece en dicha esfera, el sistema jurídico ha recurrido al reclutamiento de expertos ${ }^{6}$ especializados en el campo de las finanzas, por lo cual se ve sometido a la constante presión de la llamada

\footnotetext{
${ }^{5}$ Estrategia que también se conoce tradicionalmente como "disciplina de mercado".

${ }^{6}$ En un contexto social marcado por altos niveles de diferenciación funcional, se torna imperioso para los diversos subsistemas parciales el reclutamiento de individuos especializados en su complejidad interna, profesionales a los cuales Willke (2007) denomina expertos simbólicos.
} 
"trampa epistémica" (Teubner 2005), obstáculo en el cual el sistema jurídico se ve compelido a traducir las realidades externas dentro de sus propios parámetros cognitivos.

Dada la mixtura social que caracteriza a las estructuras regulatorias nacionales, la trampa epistémica se exacerba en comparación a lo que ocurre en los tribunales tradicionales, donde la interferencia es minimizada mediante la asistencia a soluciones procedimentales. En la jerga jurídico-financiera, circula el término de "captura regulatoria" para hacer referencia a la desdiferenciación que se genera cuando el sistema financiero consigue absorber a las agencias supervisoras dentro de su propia estructuralidad (Stigler 1971). Pagliari (2012c), sintetiza las causas de dicho proceso en la confluencia de cuatro factores primordiales: la supremacía intelectual de los profesionales en finanzas, el contexto institucional, la intervención intersistémica del aparato gubernamental, y una participación asimétrica de los stakeholders en el proceso regulatorio ${ }^{7}$.

De modo complementario, la vulnerabilidad de las superintendencias ante la desdiferenciación programática se explica a partir del concepto de "puertas giratorias" (Green 2012). La intermitente alternancia ocupacional de los reguladores entre la industria financiera y las entidades regulatorias conlleva que éstos carguen consigo la racionalidad utilitarista del subsistema financiero, lo que no se condice con la misión pública de su mandato.

Como es de esperar, la tendencia a la fusión estructural parcial (Kjaer (2011) es proclive a suceder en culturas nacionales, donde la política fiscal se encuentra orientada a extraer el máximo rendimiento del sistema financiero, para lo cual puede recurrir a su desregulación. Si se compara el grado de autonomía que poseían las entidades regulatorias en las jurisdicciones nacionales regidas por modos centralizados de coordinación sistémica (como China), en contraposición con aquellas basadas en el autogobierno (como sucede con las configuraciones regulatorias anglosajonas), éstas últimas fueron mucho más propensas a sufrir episodios de desdiferenciación dirigidos desde el sistema financiero hacia las estructu-

\footnotetext{
${ }^{7}$ La clausura operativa de los subsistemas sociales, y su ascendente complejización operativa y estructural, tornan cada vez más opacos sus procesos internos (Luhmann 2006). Esta falta de transparencia se traduce en dificultades para observar y comprender lo que acontece en los dominios funcionales vecinos, por lo que la incorporación de inversionistas en los cuerpos regulatorios, no supone un problema en sí mismo. La irrupción patológica de la racionalidad financiera en el mundo jurídico, se ejerce cuando existe un desbalance entre los stakeholders que efectivamente participan de la regulación, lo cual repercute en una inexorable captura cognitiva (2012c).
} 
ras reguladoras (Helleiner \& Pagliari 2011), como consecuencia de un estímulo proveniente desde el sistema político.

La ambivalente desdiferenciación funcional de las estructuras regulatorias a nivel nacional, coincide con el diagnóstico de Mereminskaya y Mascareño (2011) en torno a los posibles focos de colisión entre las expectativas del régimen financiero, y los intereses de las estructuras políticojurídicas nacionales.

En primer lugar, el estado puede intentar extender su control sobre las operaciones de las entidades regulatorias domésticas, corriendo el riesgo de normativizar las estructuras cognitivas del sistema financiero (Mascareño 2011), lo cual entorpece la capacidad adaptativa de dicho sistema. En segundo lugar, los mecanismos cognitivos del sistema financiero pueden estar lo insuficientemente desarrollados como para permitirle mantener su estabilidad autopoiética, lo cual se puede contener mientras la regulación fustigue la toma excesiva de riesgos. Pero cuando la programación que guía las operaciones regulatorias de las superintendencias nacionales tiende a la delegación de sus funciones al sector privado, el "riesgo moral" encuentra un terreno fértil para germinar (Dowd 2009) ${ }^{8}$, y el sistema financiero puede exteriorizar sus distorsiones a otros sistemas en su entorno.

Sin embargo, todos los elementos operativos y estructurales señalados anteriormente son susceptibles de verse modificados al ser comprendidos desde la dimensión temporal de sentido, puesto que al igual que como sucede con los ciclos autopoiéticos en el sistema económico ${ }^{9}$, la esfera jurídica orientada a la regulación también manifiesta claros patrones comunicativos en su ritmo operativo (Cross 2012), como será explicado a continuación.

Son múltiples los factores que se conjugan para detonar un incremento repentino en la actividad de las agencias regulatorias. Además de los efectos producidos por la captura regulatoria, se agrega la ocurrencia de desestabilizaciones generalizadas en los mercados financieros, las cuales gatillan un aumento súbito en el caudal de comunicaciones recursivas (Green 2012). En este sentido, existe un subyacente acoplamiento operativo entre el pulso del circuito autorreferencial financiero y la autopoiesis

\footnotetext{
${ }^{8}$ No obstante, en la crisis subprime confluyeron ambas dinámicas simultáneamente. Por una parte, el gobierno de Estados Unidos abusó de su máquina financiera, produciendo su inevitable sobrecalentamiento y respectivo cortocircuito en su hiperciclo autorreferencial (Mascareño 2011). Por otra parte, el sistema financiero fue incapaz de advertir los peligros de los instrumentos derivados de inversión (Ladeur 2011).

${ }^{9} \mathrm{O}$ dicho de manera simplificada, como sucede con el ciclo económico (Keynes 2009)
} 
del derecho ${ }^{10}$. De esta manera, la cadencia autopoiética de la regulación financiera reacciona necesariamente ante las fluctuaciones en el flujo iterativo del sistema financiero.

En términos empíricos, esto sucede por la acción agregada de un alza en los fondos disponibles para que las agencias desplieguen sus operaciones regulatorias, tal como señala Pan (2011), sumado a la presión que el sistema político ejerce para que las superintendencias enmienden la negligencia demostrada (Giadinis 2013a). Años después de disipado el desajuste generalizado de la autopoiesis financiera, el pulso regulatorio vuelve a desacelerarse hasta la aparición de una nueva debacle sistémica (Cross 2012).

En referencia a la crisis del 2008, una vez que el ciclo autopoiético financiero entró en fase de declive y la iliquidez comenzó a acechar los mercados, el ciclo regulatorio retomó su curso ascendente (Pagliari 2012a). En lo que respecta a las crisis precedentes, la respuesta gubernamental tradicional se había caracterizado por fortalecer aún más la autorregulación del sistema financiero (Gadinis 2013a). Pero esta vez fue diferente. Una pluralidad de relaciones intersistémicas nocivas fueron advertidas por las estructuras cognitivas del sistema político, cuya capacidad de aprendizaje permitió modificar sus expectativas originales y buscar nuevos métodos para asegurar la estabilidad social, lo que marcó un giro en el paradigma autorregulatorio vigente. Las razones de este vuelco intersistémico se precisan a continuación.

\section{DESDIFERENCIACIÓN DIRIGIDA ESTATALMENTE: REFORMAS REGULATORIAS DOMÉSTICAS}

Para entender algunas de las causas concretas que detonaron este quiebre, resulta relevante el análisis crítico hecho por Brasset y Clarke (2012) sobre la crisis subprime. Estos autores dejan al descubierto cómo el sistema financiero trasplantó y utilizó semánticas alusivas a catástrofes naturales tomadas desde la psicopatología, como recurso simbólico para generar una subsecuente respuesta auxiliar por parte del sistema político. Más precisamente, se difundió una verdadera "narrativa traumática" (Brasset

\footnotetext{
${ }^{10}$ Para efectos del orden social, los acoplamientos permiten la coordinación de las múltiples contingencias que dan vida a las operaciones comunicativas (Luhmann 2006), de forma tal que se vinculen en patrones definidos. Esto significa que ante determinada variación en un sistema, se puede activar una consiguiente respuesta en otro sistema, razón por la cual no se debiese confundirse coordinación con sincronización (Willke 2007).
} 
\& Clarke 2012: 14) para activar una respuesta tan veloz como onerosa de parte del Estado, el que por su parte reaccionó con abundantes inyecciones de capital a las instituciones financieras en bancarrota. De esta manera, el sistema financiero construyó astutamente la figura de una víctima desvalida (los ahorrantes) y de un codicioso perpetrador (los banqueros y los especuladores).

Gracias a dicho movimiento performativo, el sistema financiero cumplió uno de los dos objetivos perseguidos. En primer lugar, los masivos balotajes permitieron salvar a tiempo las entidades catalogadas como too big to fail, cuya insolvencia ponía en peligro a todo el sistema. A esta ayuda se agregó la participación directa de actores políticos anteriormente desligados de la regulación financiera, cuyos amplios poderes parlamentarios y ministeriales permitían agilizar el rescate sistémico (Gadinis 2013a). En segundo lugar, se perseguía desviar el foco de disrupción percibido para así no gatillar cambios estructurales significativos, sindicando como culpables a los irresponsables actores financieros y no al sistema en su conjunto. No obstante, aquella meta no pudo ser alcanzada conforme a lo esperado.

El malestar generalizado de la población en los países afectados, desataron oleadas de comunicaciones que fueron asimiladas por las estructuras democráticas de los estados. Como resultado, los actores estatales se vieron compelidos a traducir dicha insatisfacción en un mayor involucramiento de los gobiernos en las actividades regulatorias (Gadinis 2013a). Dicho de otro modo, el ingreso de los intereses de la sociedad civil al régimen financiero en medio de la vorágine gatilló un proceso de democratización relativa del régimen. En definitiva, el sistema financiero superó el umbral de tolerancia hasta el cual los gobiernos podían soportar su autodestructiva tendencia.

Con ciertas reminiscencias a lo que sucedió posteriormente a la crisis de 1929, las estructuras estatales que demostraron una excesiva permisividad financiera (y otras de modo preventivo ${ }^{11}$ ) alteraron la organización de su infraestructura regulatoria interna. El resultado se tradujo en el tránsito de una forma eminentemente cognitiva de coordinación social, basada en la mano invisible de la regulación, a un modo de coordinación regulatoria que otorga un mayor margen de discreción formal a los actores gubernamentales (Pagliari 2012a), lo que sirve de contrapeso de cara a la captura financiera de la infraestructura jurídico-regulatoria.

\footnotetext{
${ }^{11}$ De forma mimética, algunas estructuras nacionales asimilaron las medidas adoptadas por la UE con respecto a las agencias de calificación de riesgo, y a los instrumentos derivados (Pagliari 2012b).
} 
Para analizar las modificaciones centrales de la reforma regulatoria se tomarán como referencia los resultados empíricos y las valiosas conclusiones presentadas por Stavros Gadinis (2013a). En base a una muestra de quince jurisdicciones nacionales claves para el sistema financiero, dos encuestas levantadas el 2007 y el 2010 revelaron que nueve estados han amplificado la participación gubernamental en el régimen financiero, incluyendo a cuatro de los núcleos financieros más relevantes (Estados Unidos, Alemania, Francia y el Reino Unido). Sobre los países que no realizaron cambios, cuatro de ellos (Japón, Canadá, México e Italia) ya presentaban una considerable influencia estatal en su aparato regulatorio. Sólo Corea del Sur y Suiza fueron la excepción, cuyas culturas regulatorias son sumamente peculiares ${ }^{12}$.

Gadinis (2013a) se muestra enfático en mencionar que en la mayoría de las estructuras nacionales reformadas, las entidades regulatorias han conservado las funciones cognitivas de observación y descripción del entorno financiero, pero la subsecuente información generada es susceptible de ser interpretada y utilizada por agentes estatales para una posterior toma de decisiones resolutivas en torno a circunstancias cruciales. La fórmula empleada por cada gobierno para recuperar las estructuras regulatorias capturadas por el sistema financiero, ha radicado en una reestructuración del armazón institucional desde el cual emanaba dicha regulación. Más específicamente, la plataforma para su estatización indirecta se basó en dos mecanismos mutuamente imbricados.

En primer lugar, la gran mayoría de las estructuras nacionales optó por fragmentar sus entidades regulatorias en múltiples organizaciones especializadas en una de las diversas áreas del sistema financiero, lo que se tradujo en la célebre Ley Dodd-Frank para el caso de Estados Unidos, en los cuatro paquetes de reformas bancarias en Dinamarca, o en las tres actas prescritas desde el 2009 en Irlanda, por mencionar algunos de los casos estudiados por Gadinis (2013a). En concreto, los ejemplos más claros se encuentran en la abolición de la Financial Services Authority (FSA) en Gran Bretaña, cuyo aparato regulatorio se distribuyó en tres organismos con funciones más específicas, y en la respectiva creación del Financial

\footnotetext{
${ }^{12}$ En contra de la tendencia imperante, Corea del Sur contempló inicialmente la idea de introducir legislaciones desreguladoras que finalmente descartó, aunque en 2009 aprobó una reforma para incrementar la integración de las estructuras financieras sin aumentar la influencia política (Gadinis 2013a). Para el caso de Suiza, "la falta de poder ejecutivo centralizado, tales como un presidente o primer ministro" (Gadinis 2013a: 357) explicarían su desinterés por aumentar el control político sobre la industria financiera.
} 
Stability Oversight Council (FSOC) y de la Orderly Liquidation Authority (OLA) en EEUU.

En segundo lugar, la creación de nuevos aparatos regulatorios y la consolidación de los ya existentes supuso la formación de nuevos acoplamientos estructurales entre el sistema político, encarnado en la figura del Estado nación, y el sistema jurídico, representado por las entidades regulatorias. Los nuevos vínculos intersistémicos se expresaron en la creación de concilios entre los gobernadores de las diversas entidades reguladoras nacionales -incluido el representante del banco central- con un actor político como líder del consejo, o por lo menos con una considerable cantidad de miembros designados por parte del gobierno. En palabras del propio Gadinis (2013a: 368): "la toma de decisiones en estos concilios a menudo implica requisitos de supermayoría y derechos de veto que cimientan el rol primario de los políticos", lo que confiere a los actores estatales facultades para conducir las operaciones regulatorias tanto en ocasiones excepcionales, como durante el funcionamiento habitual de los mercados financieros.

Cada país que ajustó y readaptó su estructura regulatoria, lo hizo conforme a sus propios requerimientos funcionales idiosincráticos. Algunas estructuras nacionales optaron por compartir los poderes de veto entre actores gubernamentales y las agencias supervisoras, mientras que en otras el Estado posee un control total sobre los dispositivos de inyección simbólica auxiliar, es decir, sobre los rescates bancarios (Gadinis 2013a). Asimismo, a pesar de no ser la tendencia general, cuatro gobiernos optaron por "mantener más poderes indirectos que directos" (Gadinis, p.362), referidos a la designación de actores regulatorios relevantes. A grandes rasgos, el estado amplió su perímetro operativo en cuatro frentes: autoridad supervisora, aseguración de depósitos, autoridad prudencial y ejecución resolutiva.

Estos nuevos acoplamientos estructurales sirven de conducto institucional para que el estado pueda canalizar legítimamente sus irritaciones hacia el sistema jurídico, de tal forma que sus resultados operativos puedan ser concordantes con los criterios de regulación pública esperados por el gobierno. No obstante, dicha infraestructura regulatoria también puede servir como puerta de entrada a la contaminación de la autopoiesis jurídica en base a intereses puramente gubernamentales. Es posible plantear algunos escenarios en los cuales el estado podría anteponer ocasionalmente su código político elemental a la codificación binaria propia del sistema jurídico, instrumentalizando las instancias de deliberación regulato- 
ria en su propio y único beneficio funcional, lo que socavaría momentáneamente el carácter diferenciado del derecho como subsistema parcial de la sociedad ${ }^{13}$.

En lo atingente a la reconfiguración doméstica del régimen financiero, Gadinis (2013a) considera tres contextos hipotéticos en los que se podría entrever una distorsión de las operaciones jurídico-regulatorias por causas netamente políticas, recurriendo indiscriminadamente a los mecanismos de bailout.

El primero de ellos corresponde al grado de precisión temporal con el que se aprueban los rescates financieros, el cual según el autor, se podría ver afectado por el comportamiento cíclico del sistema político, definido por la ocurrencia de periodos electorales ${ }^{14}$. Gadinis (2013a) supone que, posteriormente a la ocurrencia de elecciones, los gobiernos pueden dejar caer a ciertas instituciones financieras en problemas, como consecuencia de una menor consideración sobre las preferencias de la ciudadanía. Sin embargo, el descontento generado por la pérdida de empleo, ahorros y el consecuente contagio disruptivo que conlleva el no autorizar ciertos bailouts, puede ser incluso mucho mayor que el provocado por utilizar el dinero de los contribuyentes sin su consentimiento. Por lo anterior, es poco probable que algún gobierno opte por poner en peligro la estabilidad de sus mercados financieros por una razón tan fútil como lo es la captación de popularidad a corto plazo.

El segundo foco de colisión radica en la creciente polarización de la opinión pública luego de la crisis, lo cual aumenta el riesgo de que "las elecciones de los políticos pueden reflejar eventualmente tendencias más radicales en la opinión pública" (Gadinis 2013a: 385). Nuevamente, resulta sumamente improbable que un gobierno elija encender una chispa que incendie gran parte de las operaciones financieras, al anteponer su afán por captar la simpatía del electorado. Si bien los bailouts contribuyen a crear una atmosfera propicia para el riesgo moral, los costos derivados de no facilitar el auxilio intersistémico a tiempo y en la justa proporción pue-

\footnotetext{
${ }^{13}$ Cabe destacar que la llamada desdiferenciación intersistémica, acontece con una frecuencia excepcional, ya que a ningún sistema le resulta conveniente mantener un control absoluto y sostenido de los procesos operativos de otro dominio sistémico (Mascareño 2010). En tal caso, la absorción total del sistema subordinado a manos del sistema dominante, implicaría un inevitable entorpecimiento y la consiguiente dilución de la utilidad funcional del primero, por lo cual se vuelve necesario conceder un relativo margen de autonomía, para que el segundo pueda parasitar y nutrirse de su rendimiento comunicativo (Amstutz 2011).

${ }^{14}$ Para una revisión acabada sobre cómo los procesos políticos irritan al sistema financiero, véase Carey y Lebo (2006).
} 
den ocasionar un desajuste generalizado en la operatividad de los demás subsistemas parciales, dentro y fuera de la estructura nacional en aprietos ${ }^{15}$.

En lo que respecta a la tercera consideración que podría mermar eventualmente la autonomía regulatoria del sistema jurídico, Gadinis identifica en el lobby un riesgo patente. Con la finalidad de establecer una relación de clientelismo político, el sistema financiero puede caer en prácticas que muchas veces escapan al escrutinio de la luz pública, como el costear campañas políticas ${ }^{16}$-actividad que por lo demás facilita la captura regulatoria (Pagliari 2012c), pero esta vez con el sistema político como intermediario. En este caso, la concesión de ayuda monetaria se da por hecho, quedando relegada a la contingencia la indicación de qué firmas son las que merecen ser apoyadas, y qué empresas financieras deben quebrar.

En este sentido, la verdadera cuestión es menos si rescatar o no a las entidades financieras en peligro, que en la elección de a cuáles firmas entregar su apoyo. En base a los argumentos presentados, sostengo que sólo el último caso presentado por Gadinis (2013a) constituye un ejemplo de desdiferenciación factible de ser verificado empíricamente, lo que será ilustrado tomando como ejemplo las preferencias del gobierno estadounidense durante la crisis subprime.

Cuando la crisis alcanzó su punto más álgido el año 2008, el gobierno de EEUU optó por rescatar a Fannie Mae y a Freddie Mac, dos corporaciones claves en la política pública impulsada por el gobierno de aquel entonces, las que, sin embargo, demostraron ser responsables de tomar un excesivo riesgo en la concesión de hipotecas (Mascareño 2011). La correspondencia de los servicios ofrecidos por ambas entidades con los intereses estatales, y su gran capacidad de influencia política, les valió un amplio favoritismo que se tradujo en un cuantioso préstamo otorgado con el dinero de los contribuyentes, como queda expresado por Richard Painter:

En el mercado hipotecario, las empresas patrocinadas por el gobierno (GSE), más notablemente Freddie Mac y Fannie Mae, contribuyeron significativamente

\footnotetext{
${ }^{15}$ Lo que se evidencia en el caso de Japón en la década de los 90. Los efectos negativos de no haber decidido intervenir a tiempo sus bancos afectados, se han manifestado en una severa y persistente deflación.

${ }^{16}$ Como se demostró en la condonación de las deudas sostenidas por el Partido Socialista Obrero Español con el Banco Santander y con la caja de ahorros de Bilbao y Vizkaya en el 2012, por doce y veintiún millones de euros respectivamente, durante el gobierno de aquel partido político (Hernanz 2011); (Elmundo.es 2006).
} 
al nivel de riesgo y usaron influencias políticas y hábil cabildeo para impedir los esfuerzos para impedir sus actividades (Painter 2009: 134).

De igual forma, otras firmas recibieron un apoyo similar de parte del Estado. No obstante, el célebre banco de inversión Lehman Brothers no corrió la misma suerte, desplomándose de forma espectacular en septiembre de 2008. Los criterios que guían a los actores políticos a rescatar a ciertas firmas y no a otras pueden levantar suspicacias cuando existe una ausencia de estructuras protocolares adecuadas que transparenten aquella toma de decisiones:

No hay reglas jurídicas preestablecidas o instituciones para implementar las normas. Funcionarios políticos toman la mayoría de esas decisiones, y a veces esas decisiones pueden parecer arbitrarias (por ejemplo, Bear Stearns y AIG son rescatados, pero Lehman Brothers no). Cuando conflictos de interés y otros problemas éticos contribuyen a la arbitrariedad en las decisiones gubernamentales con respecto a rescates extraordinarios, los funcionarios pueden malgastar el dinero público, erosionando la confianza pública en el gobierno. (Painter 2009: 132).

Para evitar interpretaciones sesgadas de lo que se busca definir como lobby en este texto, cabe precisar que la ciudadanía también puede irritar a las estructuras estatales a través de acciones colectivas o movimientos sociales. Actores pertenecientes al campo financiero y otros fuera de dicha esfera, también pueden vincularse políticamente con la finalidad de intentar adaptar la regulación a sus aspiraciones. De hecho, algunas medidas gubernamentales emprendidas para enfrentar los vicios regulatorios han sido contrarrestadas por la acción de la industria financiera (Pagliari 2012a).

Como se mencionó supra, el sistema político posibilita el acceso a sus operaciones comunicativas tanto a agentes estatales como a actores privados, por lo que resulta habitual la formación de coaliciones entre las instituciones financieras para ejercer presión contra cierta disposición regulatoria, ya sea a nivel doméstico, regional y transnacional. No obstante, también existe una táctica de lobby subvalorada por la literatura académica especializada. Pagliari y Young (2013), presentan evidencias que confirman la participación de una heterogeneidad de instituciones extrafinancieras en los procesos de resistencia regulatoria. Para ejercer un feedback negativo sobre las pretensiones del sistema jurídico, el sistema financiero no sólo moviliza políticamente a sus diversos stakeholders sec- 
toriales, sino que además se agregan stakeholders pertenecientes al sistema de la economía real ${ }^{17}$. Empresas representativas de rubros como la agricultura, las telecomunicaciones o la energía, pueden aceptar agruparse en bloques junto a determinada industria financiera, dado que medidas más restringentes a la oferta de crédito disponible, pueden perjudicar sus planes de negocios.

De todos modos, la reflexividad del sistema jurídico le ha permitido activar ciertos mecanismos de defensa que dificulten la desdiferenciación ${ }^{18}$. Gadinis (2013a)menciona que en EEUU la legislación contempla la apelación a tribunales en caso de decisiones arbitrarias por parte del FSOC, así como exige una justificación formal del Tesoro de su Majestad para respaldar ciertas resoluciones claves para el caso de Gran Bretaña. No obstante, en última instancia, la existencia de tales resguardos representa un resguardo más formal que práctico.

Sin embargo, las entidades regulatorias también han amplificado su rango de intervención supervisora. Pagliari (2012b 2012c) ha evidenciado una extensión del alcance regulatorio doméstico y regional, en los campos financieros ajenos a la fiscalización jurídica previamente a la crisis ${ }^{19}$, los cuales surgieron como motivo del llamado boom de la innovación financiera desde el último cuarto del siglo $X X^{20}$. Sobre lo mismo, la pluralidad de cambios acontecidos en los niveles inferiores y medios del régimen financiero global estimularon la adaptación de su escalafón superior al nuevo estado de complejidad emergente. Más específicamente, tanto la estatización de la regulación doméstica como los cambios en el statu quo en el plano internacional ${ }^{21}$, han redundado en la formación de nuevos acoplamientos estructurales en la infraestructura institucional transnacional, y en

\footnotetext{
${ }^{17}$ Pero los stakeholders extra-económicos también pueden agruparse políticamente, y presionar en contra de los intereses de las instituciones sujetas a la regulación (Pagliari \& Young 2013).

${ }^{18}$ De todas maneras, Schultz y Thompson (2011) ofrecen una perspectiva que pone en entredicho la supuesta autonomía de los reguladores domésticos. Ya que en la práctica, las actividades regulatorias deben rendir cuentas e ir de la mano con las políticas gubernamentales.

${ }^{19}$ Las medidas apuntadas a cubrir las lagunas regulatorias que surgieron producto de la acelerada complejización estructural del sistema financiero, se han enfocado en el mercado en las sombras (OTC), las agencias de calificación de riesgo y los fondos libres de inversión.

20 Sobre esta verdadera explosión de nuevos artilugios técnicos para el despliegue de las operaciones financieras, véase Best (2010).

${ }^{21}$ Uno de los efectos más sorpresivos de la crisis del 2008, se encuentra en el debilitamiento de la distinción centro/periferia presente en el contexto internacional (Kjaer 2011), referido específicamente a la disminución de la brecha existente entre las estructuras nacionales desarrolladas, y aquellas en vías de desarrollo. El principal vestigio que confirma esta dinámica radica en la conformación del G20 como principal foro de cooperación económica internacional, lo que ha permitido incluir la perspectiva de numerosas culturas nacionales -otrora excluidas- a la institucionalidad existente.
} 
una correspondiente reprogramación de los principios que guían la producción de sus estructuras normativas.

A pesar de la gran responsabilidad funcional que poseía el Financial Stability Forum (FSF), sus ambiguas metas e incipientes resultados demostraron ser un obstáculo para afrontar los escollos regulatorios que reveló la crisis, lo que motivó su remodelación en abril del 2009. Como resultado, el FSF fue transformado en el Financial Stability Board (FSB), con una matriz de planificación robusta y con una expansión de su membrecía acorde a la creación del G20 (BIS 2013). De este modo, se constituyó como el auténtico cuartel general de la regulación financiera global, aunque sólo para alcanzar ejes de compatibilidad normativa débil entre los sistemas funcionales que allí convergen (Teubner 2005), sin la capacidad para tomar e imponer resoluciones punitivas.

La modificación del diseño organizacional del FSB suministró las condiciones necesarias para generar un nuevo acoplamiento estructural entre el sistema jurídico y el sistema político, representado por las estructuras estatales y sus actores gubernamentales, quienes integran el plenario del organismo regulatorio. Por medio esta instancia, los ministros de finanzas de las estructuras nacionales más relevantes del G20 han canalizado sus inquietudes regulatorias y moldeado las estructuras normativoprocedimentales acordadas en el FSB, cuya punta de lanza radica en una serie de marcos regulatorios dirigidos a enmendar el desperfecto que significan las entidades Too-Big-To-Fail (TBTF) (FSB 2013), de manera tal que se pueda terminar -o por lo menos atenuar- el parasitismo intersistémico entre sistema financiero y sistema político, provocado por los bailouts.

\section{CONCLUSIONES}

Si bien han sido numerosos los avances evolutivos experimentados por el régimen financiero en los últimos cinco años, no se han desmantelado los rasgos centrales del esquema regulatorio previo a la crisis, persistiendo la disciplina de mercado como el principal mecanismo de autorregulación. Por lo mismo, las medidas adoptadas están lejos de tener un impacto equiparable a las respuestas estatales tomadas con posterioridad a la gran crisis de 1929. Aunque resulta también evidente que muchos efectos de la 
crisis del 2008 traen reminiscencias de aquel fatídico evento ${ }^{22}$. De todos modos, las transformaciones de la arquitectura financiera global revisadas en el presente artículo expresan la ocurrencia de un genuino momento constitucional $^{23}$, cuyo despliegue está impelido por el sistema político pero codificado en base a construcciones normativas que demuestran compatibilidad con la racionalidad financiera, lo cual no sería posible sin la habilidad reflexiva del derecho.

Como es de esperar, la extensión temporal de este proceso queda determinada por el advenimiento del cénit del ciclo regulatorio. Debido a la iteración episódica de la autopoiesis jurídica acoplada a la operatividad financiera, es difícil anticipar si los marcos regulatorios impulsados podrán sostenerse considerablemente en el futuro, o si serán efectivamente rechazados por el sistema financiero a través de irritaciones negativas. Y por lo visto, así seguirá siendo mientras el régimen cuente con una mala memoria, insuficiente capacidad de aprendizaje a largo plazo, y recurra al empleo de medidas más reactivas que preventivas.

Conforme a lo presentado en el desarrollo de este artículo, queda en evidencia la escaza efectividad de la doble reflexividad del sistema jurídico, capacidad mediante la cual éste puede generar normas para limitar la misma producción de normas para así producir anticuerpos que impidan su captura definitiva por parte del sistema financiero. Cada cierto intervalo temporal, esta condición redunda en la intensificación del control políticoestatal en la infraestructura regulatoria nacional y extra-doméstica, lo que merma el rango de discrecionalidad operativa del sistema jurídico desde dos direcciones funcionales, las que, contrario a lo que pudiese pensarse, no son del todo irreconciliables.

Como lo demuestran las reformas efectuadas para corregir los vacíos regulatorios del régimen, pueden coexistir simultáneamente férreos mecanismos de control político con la captura de las bases regulatorias de la esfera jurídica, dado que las necesidades de apoyo cognitivo que manifiesta el régimen financiero a escala doméstica (y transnacional) no se solucionan con la simple creación de mayores dispositivos de fiscalización. De hecho, si se buscase extirpar definitivamente la captura regulatoria,

\footnotetext{
${ }^{22}$ Como se constata en la progresiva y generalizada adopción de barreras proteccionistas a nivel internacional, tanto para las inversiones como para el comercio de bienes y servicios (Borak 2013), por mencionar un ejemplo.

${ }^{23}$ Como aduce Teubner (2011), corresponde a un evento de auto-conservación que se activa cuando la autodestrucción del subsistema parcial se torna inminente: "The functionally-differentiated society appears to ignore earlier opportunities for self-correction [...] The endogenous self-energising processes are so dominant that they allow self-correction only at the very last moment." (p.11).
} 
habría que advertir que el problema no sólo reside en una desdiferenciación programática y estructural ejercida desde el dominio financiero porque, hasta cierto punto, aquellos rasgos sistémicos son los suficientemente genéricos como para permitir su similitud y convergencia entre diversos sistemas sociales ${ }^{24}$. El desafío real está en cómo procedimentalizar las actividades de los expertos simbólicos que se insertan desde el sistema financiero al sistema jurídico, de tal manera que su cosmovisión sea congruente con las operaciones regulatorias exigidas.

Por lo mismo, el derecho financiero pareciera presentarse al sistema jurídico como una entidad inerte, incapaz de defenderse de la lucha que establecen los dos subsistemas sociales mencionados. Pero, de cierta manera, la permisividad demostrada por el sistema jurídico en esta dinámica ha sido requisito para que las estructuras nacionales con su regulación financiera "capturada", hayan podido explotar al máximo el rendimiento operativo del sistema financiero, nutriendo al resto de los subsistemas sociales de sus beneficios funcionales. Claro está, los daños se dejan sentir con la ocurrencia de cada crisis, recordando intermitentemente que todo sistema posee límites ineludibles.

Pero es precisamente en aquellas instancias cuando interviene el sistema político, como contrapeso a los excesivos y recurrentes tropiezos financieros. En este sentido, independientemente de la momentánea pérdida de autonomía que experimenta el sistema jurídico como consecuencia de la desdiferenciación, su necesaria variabilidad estructural se activa y dirige en función de la irritación que recibe alternadamente de ambos subsistemas parciales, por lo que si bien este proceso dialéctico ha sido catalogado como eminentemente disfuncional, sus ventajas relucen cuando se le observa dentro de márgenes temporales lo suficientemente amplios $^{25}$. Dicho de otro modo, el doble movimiento descrito a lo largo de este artículo constituye un claro reflejo de los patrones que sigue la evolución social en ciertas circunscripciones territoriales, cuya idiosincrasia apuesta por la adaptación mutua de los tres subsistemas parciales "partícipes" de la regulación financiera, más basada en la persecución de -a ve-

\footnotetext{
${ }^{24}$ Es más fácil hacer coincidir estructuras y programas, que racionalidades o realidades epistémicas. Algunas estructuras sirven de bisagra entre diversos subsistemas sociales, las cuales justamente permiten el acoplamiento estructural entre ellos. Asimismo, pueden encontrarse programaciones relativamente comunes entre diversos sistemas parciales, respetando su código elemental claro está.

${ }^{25}$ A pesar de que en esta reflexión se indagaron los efectos de una reforma institucional, la misma utilidad estabilizadora de la desdiferenciación podría ser identificada en eventos críticos como revoluciones o golpes de estado, cuyo objetivo inmediato sea contrarrestar la sobre-extensión de algún subsistema parcial, creciendo más allá de los límites que aseguran su integridad y la de su entorno.
} 
ces transgresoras- estrategias de interferencia y de ajuste mutuo, que en la imposición centralizada de iniciativas autoritarias de coordinación. RM

\section{REFERENCIAS}

Amstutz, M. (2011). Eroding Boundaries on Financial Crisis and Evolutionary Concept of Regulatory Reform. En P. Kjaer, G. Teubner, \& A. Febbrajo (Eds.), The Financial Crisis in Constitutional Perspective: The Dark Side of Functional Differentiation. (pp. 224-268). Oxford: Hart Publishing.

Armijo, L. (2001). The Political Geography of World Financial Reform: Who Wants What and Why? Global Governance, 7(4), 379-396.

Best, J. (2010). The Limits of Financial Risk Management: Or what we didn't learn from the Asian Crisis. New Political Economy, 15(1), 29-49.

Borak, D. (2013). Financial Stability Board Warn Countries Against Protectionism. American Banker, 178(61), 25.

Brasset, J., \& Clarke, C. (2012). Performing the Sub-Prime Crisis: Trauma and the Financial Event. International Political Sociology, 6(1), 4-20.

Carey, S., \& Lebo, M. (2006). Election Cycles and the Economic Voter. Political Research Quarterly, 59(4), 543-556.

Cross, G. (2012). Addressing the Problem of Cyclical Capture. En S. Pagliari (Ed.), The Making Good Financial Regulation (pp. 173-180). Londres: International Centre for Financial Regulation.

Douglas, A., \& Taylor, M. (2009). The Global Financial Crisis and the Financial Stability Board: Hardening the Soft Law of International Financial Regulation? AIIFL Working Paper, 32(6), 488-513.

Dowd, K. (2009). Moral Hazard and the Financial Crisis. Cato Journal, 29(1), 141-166.

Febbrajo, A. (2011). The Failure of Regulatory Institutions? En P. Kjaer, G. Teubner, \& A. Febbrajo (Eds.), The Financial Crisis in Constitutional Perspective: The Dark side of Functional Differentiation (pp. 269-302). Oxford: Hart Publishing.

FSB (2013). Progress and Next Steps towards Ending "Too-Big-To-Fail" (TBTF). Report of the financial Stability Board to the G20.

Gadinis, S. (2013a). From Independence to Politics in Financial Regulation. Berkeley Law Scholarship Repository, 327(101), 327-389.

Gadinis, S. (2013b). The Financial Stability Board: The New Politics of International Financial Regulation. Texas International Law Journal, 48(2), 157-176.

Green, D. (2012). Political Capture and the Regulatory Cycle. En S. Pagliari (Ed.), The Making Good Financial Regulation (pp. 136-148). Londres: International Centre for Financial Regulation.

Helleiner, E., \& Pagliari, S. (2011). The End of an Era in International Financial Regulation? A Postcrisis Research Agenda. International Organization, 65(1), 169200.

Keynes, J. (2009). Teoría general de la ocupación, el interés, y el dinero. México D.F.: Fondo de Cultura Económica.

Kjaer, P. (2011). Law and Order within and Beyond National Configurations. En P. Kjaer, G. Teubner, \& A. Febbrajo (Eds.), The Financial Crisis in Constitutional 
Perspective: The Dark side of Functional Differentiation (pp. 395-430). Oxford: Hart Publishing.

Ladeur, K. (2011). The Financial Market Crisis-A Network Failure?. En P. Kjaer, G. Teubner, \& A. Febbrajo (Eds.), The Financial Crisis in Constitutional Perspective: The Dark side of Functional Differentiation (pp. 63-92). Oxford: Hart Publishing.

Lo Schiavo, G. (2013). The European Supervisory Authorities: A True Evolutionary Step along the Proccess of European Financial Integration? En N. Bukantenié (Eds.), The Interaction of National Legal Systems: Convergence or Divergence? (pp. 293-301). Vilnius: Vilnius University.

Luhmann, N. (2005). El derecho de la sociedad. México D.F.: Editorial Herder. Luhmann, N. (2006). La sociedad de la sociedad. México D.F.: Editorial Herder.

Mascareño, A. (2007). Regímenes jurídicos en la constitución de la sociedad mundial. Política Criminal, 4, 1-39.

Mascareño, A. (2010). Diferenciación y contingencia en América Latina. Santiago de Chile: Ediciones Universidad Alberto Hurtado.

Mascareño, A. (2011). Crisis Ethics. En P. Kjaer, G. Teubner, \& A. Febbrajo (Eds.), The Financial Crisis in Constitutional Perspective: The Dark side of Functional Differentiation (pp. 333-354). Oxford: Hart Publishing.

Mascareño, A. (2013). Justicia global y justicia sectorial en la sociedad mundial. Momentos de universalidad en la lex mercatoria. Dilemata, 5(13), 45-68.

Mereminskaya, E., \& Mascareño, A. (2005). La desnacionalización del derecho y la formación de regímenes globales de gobierno. En M. Martinic (Ed.), Sesquicentenario del Código Civil de Andrés Bello: Pasado, presente y futuro de la codificación (pp. 1391-1427). Santiago: Lexis-Nexis.

Mereminskaya, E., \& Mascareño, A. (2011). Colisión y armonización de regímenes regulatorios en contextos sociales globales. Fondecyt $\mathrm{N}^{\circ} 1110437$.

Pagliari, S. (2012a). Who Governs Finance? The Shifting Public-Private Divide in the Regulation of Derivatives, Rating Agencies and Hedge Funds. European Law Journal, 18(1), 44-61.

Pagliari, S. (2012b). A Wall around Europe? The European Regulatory Response to the Global Financial Crisis and the Turn in Transatlantic Relations. Journal of European Integration, 35(4), 391-408.

Pagliari, S. (2012c). How Can We Mitigate Capture in Financial Regulation? En S. Pagliari (Ed.), The Making Good Financial Regulation (pp. 1-59). Londres: International Centre for Financial Regulation.

Pagliari, S., \& Young, K. (2013). Leveraged Interests. Financial Industry Power and the Role of Private Sector Coalitions. Review of International Political Economy, 21(3), 575-610.

Painter, R. (2009). Bailouts: An Essay on Conflicts of Interest and Ethics When Government Pays the Tab. Minnesota Legal Studies Research Paper, N 09/37.

Pan, E. (2011). Understanding Financial Regulation. Cardozo Legal Studies Research Paper, No. 329, 1897-1947.

Polanyi, K. (2001). La gran transformación. México D.F.: Fondo de Cultura Económica. Schultz, L., \& Thompson, R. (2010). The Future of Agency Independence. Vanderbilt Law Review, 63(599), 623-646. 
Seeling, S., \& Novoa, A. (2009). Governance Practices at Financial Regulatory and Supervisory Agencies. IMF Working Paper.

Stigler, G. (1971). The Theory of Economic Regulation. The Bell Journal of Economics and Management Science, 2(1), 3-21.

Teubner, G. (2005). El derecho como sistema autopoiético de la sociedad global. Bogotá: Universidad externado de Colombia.

Teubner, G. (2011). A Constitutional Moment? The Logics of 'Hitting the Bottom'. En P. Kjaer, G. Teubner, \& A. Febbrajo (Eds.), The Financial Crisis in Constitutional Perspective: The Dark side of Functional Differentiation (pp. 3-42). Oxford: Hart Publishing.

Teubner, G. (2012). Constitutional Fragments: Societal Constitutionalism and Globalization. Oxford: Oxford University Press.

Stäheli, U. (2011). Political Epidemiology and the Financial Crisis. En P. Kjaer, G. Teubner, \& A. Febbrajo (Eds.), The Financial Crisis in Constitutional Perspective: The Dark side of Functional Differentiation (pp. 113-130). Oxford: Hart Publishing.

Willke, H. (2007). Smart Governance: Governing the Global Knowledge Society. Chicago: The University of Chicago Press.

\section{FUENTES DE INTERNET}

BIS (2013). Financial Stability Forum decides to broaden its membership. Consultado el 4 de junio de 2013. http://www.bis.org/press/p090312c.htm.

Elmundo.es (2006). "La BBK perdona al PSOE 21 millones de euros tras cancelar una deuda impagada desde los 80". Consultado el 10 de septiembre de 2013. http://www.elmundo.es/elmundo/2006/06/09/espana/1149820855.html

Hernanz, C. (2011). "Santander 'refinanció' la deuda del PSOE y dio una hipoteca a Zapatero durante su mandato". Consultado el 10 de septiembre de 2013. http://www.elconfidencial.com/economia/2011/11/28/santander-refinancio-ladeuda-del-psoe-y-dio-una-hipoteca-a-zapatero-durante-su-mandato-88466/

SOBRE EL AUTOR

Benjamín Sánchez Cobo es sociólogo por la Universidad Alberto Hurtado, Chile y Bachiller en Humanidades por la misma universidad. Actualmente es consultor para la Facultad de Ciencias Sociales de la Universidad Andrés Bello, Chile, sobre políticas públicas comunales y uso del tiempo urbano.

CONTACTO: bscobo@gmail.com

Recibido: Diciembre 2014

Aceptado: Abril 2015 